\title{
Comunicação
}

[Communication]

\section{Cor triatriatum sinister e hipertensão arterial pulmonar secundária em cão}

\author{
[Cor triatriatum sinister and secondary pulmonary arterial hypertension in a dog] \\ T. Champion ${ }^{1}$, F.N. Gava ${ }^{2}$, E. Garrido ${ }^{2}$, A.L.B. Galvão ${ }^{2}$, A.A. Camacho ${ }^{2}$ \\ ${ }^{1}$ Universidade de Vila Velha-Vila Velha, ES \\ ${ }^{2}$ Universidade Estadual Paulista Júlio de Mesquita Filho - FCAV/Unesp - Jaboticabal, SP
}

\begin{abstract}
Cor triatriatum refere-se à subdivisão do átrio por uma membrana fibromuscular em duas câmaras: proximal e distal. Classicamente, a câmara proximal, ou superior, recebe o sangue das veias pulmonares no átrio esquerdo e das veias cavas no átrio direito, e a câmara distal, ou inferior, está em contato com a valva atrioventricular. Trata-se de uma enfermidade congênita rara, de origem embriológica ainda não muito bem esclarecida (Kittleson, 1998; Almeida et al., 2012). As descrições em cães referem-se sobretudo ao átrio direito (cor triatriatum dexter) (Tobias et al., 1993; Mitten et al., 2001; Tanaka et al., 2003). Nessa situação, a correção pode ser realizada por meio de cirurgia com inflow occlusion (Mitten et al., 2001), ou por dilatação com balão sem necessidade de circulação extracorpórea (Adin e Thomas, 1999; Johnson et al., 2004) ou, ainda, com atriotomia e circulação extracorpórea (Tanaka et al., 2003).
\end{abstract}

A ocorrência dessa anomalia em átrio esquerdo (cor triatriatum sinister) foi relatada em cães, apesar de ser mais comum em gatos (Tobias et al., 1993; Kittleson, 1998; Almeida et al., 2012). A gravidade dos casos depende de vários fatores, como a presença de outras anomalias congênitas associadas, do tamanho e da forma da membrana que separa o átrio (Kittleson, 1998; Oliveira et al., 2011; Almeida et al., 2012).

A embriogênese dessa anomalia não está bem esclarecida e existem apenas algumas teorias que especulam a respeito. No embrião existe a veia pulmonar comum, que surge a partir do broto pulmonar, localizando-se na região superior da parede atrial esquerda. No desenvolvimento embrionário normal, existe a absorção desse tecido pela parede atrial com a formação de canais de

Recebido em 16 de novembro de 2012

Aceito em 1 de outubro de 2013

E-mail: tatiana.champion@gmail.com drenagem que irão formar as veias pulmonares. Quando essa absorção não ocorre, não há incorporação da circulação pulmonar no átrio esquerdo, gerando uma membrana que separa o átrio esquerdo em duas cavidades (teoria da máincorporação). Outra teoria refere-se a um crescimento anormal do septum primum (teoria da má-septação). Há também uma teoria em que o corno direito do seio venoso do embrião se liga à veia pulmonar comum e impede sua incorporação no átrio esquerdo (teoria do "encarceramento") (Nassar e Hamdan, 2007).

O diagnóstico do cor triatriatum é realizado por meio de ecocardiograma, mas também podem ser empregados exames de tomografia computadorizada, ressonância magnética e ecocardiografia em três dimensões (3D) (Thakrar, 2007; Almeida, 2012).

O presente trabalho relata a ocorrência de cor triatriatum sinister em um cão da raça Poodle, com hipertensão arterial pulmonar associada à insuficiência cardíaca congestiva esquerda.

Foi atendido no Hospital Veterinário "Governador Laudo Natel da FCAV-UNESP- Jaboticabal", um cão, poodle, fêmea, com cinco anos, com histórico de intolerância ao exercício e dispneia expiratória aguda. Ao exame clínico, apresentava cianose de membranas mucosas, posição ortopneica e crepitação pulmonar. À ausculta cardíaca, demonstrava bulhas normofonéticas, ritmo taquicárdico e regular e ausência de sopro. O animal foi atendido prontamente devido ao quadro emergencial, sendo medicado com furosemida na

dose de $4 \mathrm{mg} / \mathrm{kg}$ por via intravenosa e mantido em 
oxigenoterapia. No entanto, não houve êxito na estabilização do paciente e não foi possível a realização de exames complementares. $\mathrm{O}$ animal apresentou insuficiência renal aguda anúrica, parada cardiorrespiratória e óbito agudamente.

O cão foi encaminhado para exame necroscópico, o qual revelou, à macroscopia, uma membrana distinta, que dividia o átrio esquerdo em duas câmaras, cuja avaliação histopatológica revelou padrão morfológico fibromuscular, compatível com o diagnóstico de cor triatriatum sinister (Fig. 1).
$\mathrm{O}$ átrio esquerdo recebia a drenagem venosa pulmonar em sua câmara proximal, e, na câmara distal, localizavam-se a aurícula esquerda e a valva mitral. Por sua vez, a valva mitral não revelou alterações histopatológicas, mostrando a conformação histopatológica típica.

Constatou-se ainda edema pulmonar e espessamento acentuado da túnica média da artéria pulmonar, indicando hipertensão arterial pulmonar (Fig. 2), congestão passiva hepática e degeneração tubular renal.

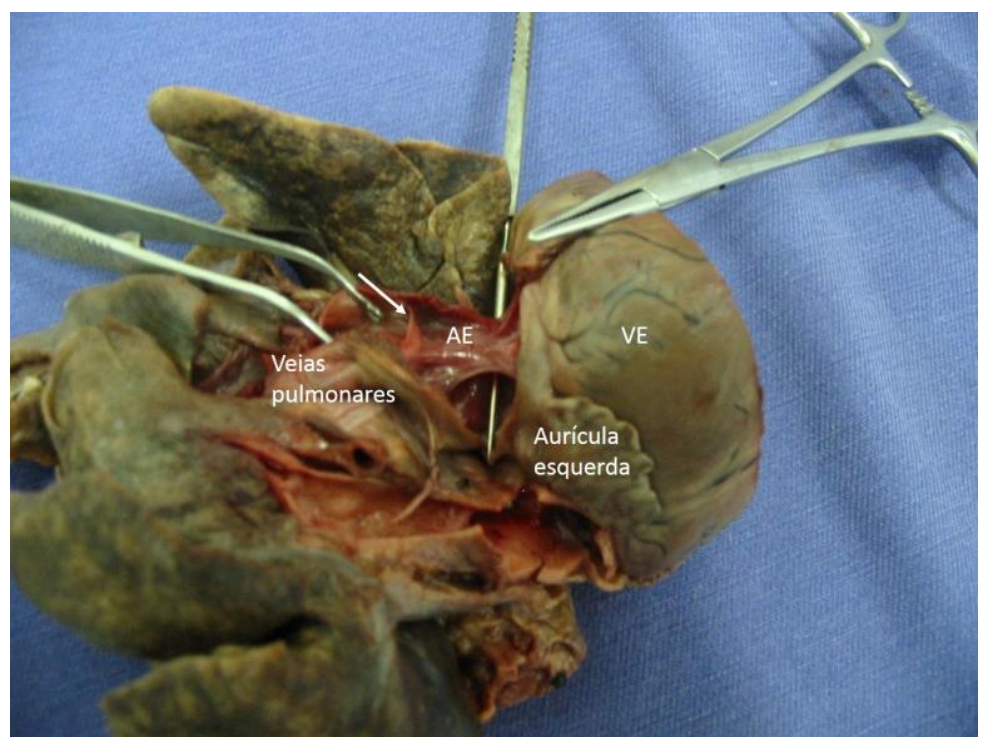

Figura 1. Imagem macroscópica de coração de cão, demonstrando membrana fibromuscular (seta) subdividindo o átrio esquerdo em duas câmaras, inferior e superior, caracterizando cor triatriatum sinister. AE representa o átrio esquerdo com cor triatriatum sinister e VE representa o ventrículo esquerdo.
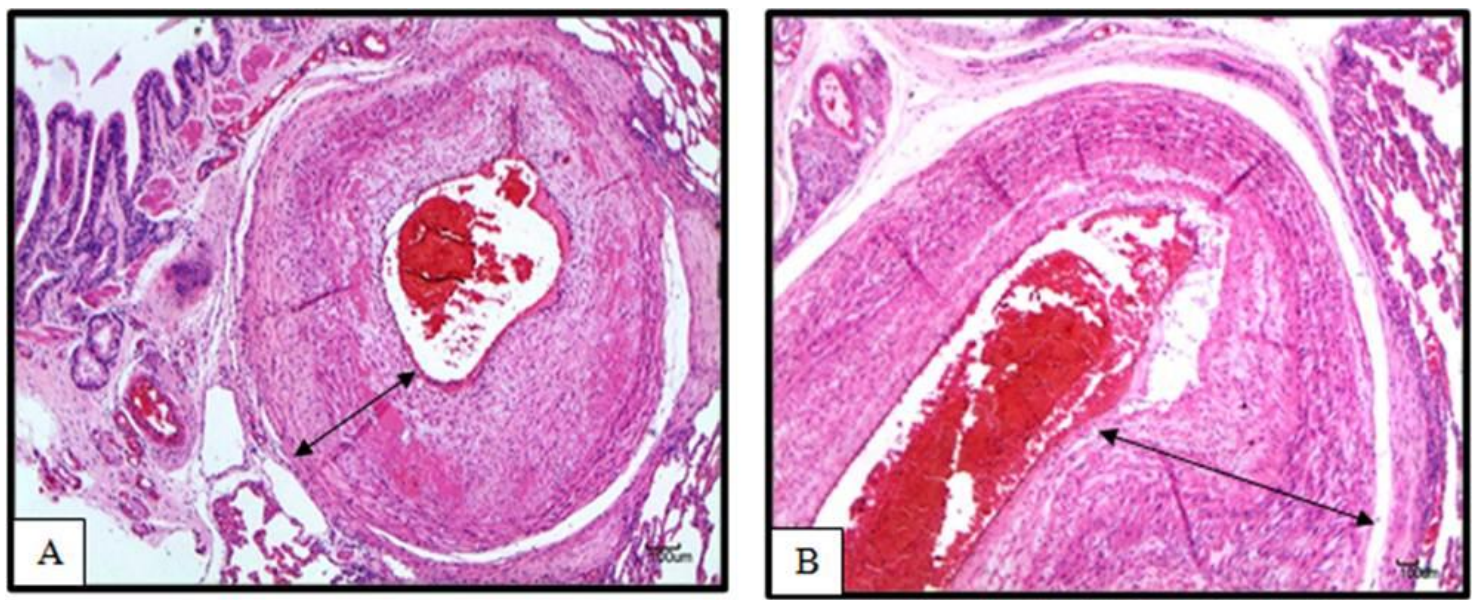

Figura 2. Corte histológico transversal (A) e longitudinal (B) de arteríolas pulmonares, demonstrando espessamento da túnica média (setas), coloração HE, Bar= 100 um. 
O diagnóstico de cor triatriatum sinister foi realizado por meio de exame patológico, cuja macroscopia evidenciou uma membrana localizada no átrio esquerdo, posicionada em região distinta à da inserção da valva mitral. Essa é uma afecção cardíaca congênita rara em cães, sendo mais frequente a ocorrência do lado direito (cor triatriatum dexter) nessa espécie (Tobias et al., 1993; Kittleson, 1998; Almeida et al., 2012).

A má-formação do sistema venoso pulmonar durante o desenvolvimento do sistema venoso fetal resulta em prejuízo do retorno venoso, podendo culminar com edema pulmonar e aumento da resistência pulmonar, gerando hipertensão pulmonar e cor pulmonale (Kittleson, 1998), conforme verificado no caso clínico descrito. A incorporação do sistema venoso pulmonar fetal no átrio esquerdo é prejudicada, levando à formação de uma câmara proximal que subdivide o átrio. A parede comum entre essas câmaras geralmente é espessa e fibromuscular, conforme descrito nos achados histopatológicos do caso clínico exposto.

Em medicina veterinária, há descrição de cor triatriatum em animais com idade variando de meses a 15 anos, cujo diagnóstico ocorre após a apresentação de quadros de insuficiência cardíaca congestiva (Oliveira et al., 2011). O animal foi apresentado para atendimento em quadro clínico de insuficiência cardíaca grave e o baixo débito cardíaco que, associado ao uso de furosemida, podem ter precipitado o desenvolvimento de necrose tubular aguda, contribuindo para o agravamento do quadro clínico.

Não foram realizados exames diagnósticos complementares, como ecocardiografia, devido à instabilidade do quadro respiratório do animal, uma vez que a contenção mecânica poderia agravar a disfunção respiratória. Nesse caso, exames não invasivos, como ecocardiografia, poderiam definir o diagnóstico antemortem de cor triatriatum sinister e talvez estimar a pressão da artéria pulmonar, definindo a hipertensão arterial pulmonar (Kittleson, 1998).

Com a separação do átrio esquerdo em duas câmaras, as veias pulmonares drenam para a câmara anterior, e a câmara posterior está em contato com a valva mitral. Uma membrana constituída de pequenas fenestras gera um aumento na resistência do fluxo sanguíneo, promovendo aumento de pressão na câmara anterior esquerda e, consequentemente, nas veias e capilares pulmonares, gerando edema pulmonar. Como ocorre aumento na resistência no leito pulmonar, ocorre aumento da pressão no tronco da artéria pulmonar, levando a um quadro de hipertensão arterial pulmonar e cor pulmonale (Kittleson, 1998).

Os achados de exame físico variam perante o diagnóstico de cor triatriatum dexter ou sinister. Animais com diagnóstico de cor triatriatum sinister podem apresentar sinais de insuficiência cardíaca esquerda, e, em seres humanos, cita-se o desenvolvimento de insuficiência cardíaca direita devido à hipertensão pulmonar e cor pulmonale. Pacientes podem apresentar sopros cardíacos apenas em alguns casos, dependendo do tamanho do orifício entre as câmaras e da intensidade do fluxo transmembrana. Geralmente, quando se ausculta sopro em filhotes com cor triatriatum, deve-se investigar outra anomalia congênita concomitante (Kittleson, 1998; Oliveira et al., 2011). Os achados eletrocardiográficos também dependem da gravidade do quadro e em geral apresentam-se sem alterações. Podem estar presentes: ritmo sinusal, complexos atriais prematuros, desvio de eixo, sobrecargas atriais e/ou ventriculares, principalmente nos casos em que há cor pulmonale (Almeida et al., 2012).

O ecocardiograma consiste na ferramenta mais importante para o diagnóstico do cor triatriatum em medicina veterinária. A membrana fibromuscular que divide o átrio em duas câmaras pode ser visualizada nos cortes: paraesternal direito transversal ou longitudinal e também no apical esquerdo. Como essa membrana pode criar uma obstrução para o fluxo sanguíneo, ocorre um gradiente de pressão entre as duas câmaras que pode ser visualizado e quantificado com o uso do Doppler de fluxo. Um fluxo turbulento também pode ser visto com o uso do Doppler em cores na região da membrana. O ecocardiograma também é útil na identificação de outras doenças congênitas associadas e no diagnóstico da hipertensão pulmonar, através da estimativa da pressão no tronco da artéria pulmonar. Os sinais de cor pulmonale, como dilatação e hipertrofia de ventrículo direito, também podem ser diagnosticados com o uso dessa técnica (Boon, 1998; Thakrar et al., 2007). 
O cor triatriatum sinister deve ser diferenciado de outras más-formações que envolvem o lado esquerdo do coração, sobretudo com estenose valvar mitral. No entanto, os aspectos macroscópicos e microscópicos da valva mitral do caso exposto permitem a exclusão desse diagnóstico diferencial (Almeida et al., 2012).

O cor triatriatum compreende uma má-formação congênita rara, que tem sido descrita em humanos, gatos e cães. $\mathrm{O}$ prognóstico da enfermidade varia de favorável a reservado, dependendo das alterações hemodinâmicas originadas pela presença da membrana fibromuscular que divide o átrio em duas câmaras. É relevante salientar o conhecimento a respeito da doença, principalmente em pacientes com sinais de doença cardíaca e ausência de sopros audíveis, pois muitas vezes o diagnóstico de cor triatriatum não é realizado precocemente.
O diagnóstico precoce pode permitir a correção definitiva e, geralmente, os cães atingem idade adulta sem alterações subsequentes (Adin e Thomas, 1999; Mitten et al., 2001).

No paciente desta comunicação, o estágio avançado de descompensação respiratória e hemodinâmica associado ao quadro de necrose tubular agravaram o prognóstico, não permitindo intervenções clínicas e terapêuticas corretivas. Ademais, ocorrência de hipertensão arterial pulmonar é raramente descrita em seres humanos, sobretudo com diagnóstico tardio em indivíduos adultos (Akintunde, 2011), e o presente relato descreve a ocorrência dessa complicação associada à insuficiência cardíaca congestiva esquerda em cão adulto.

Palavras-chave: cardiopatia congênita, átrio esquerdo, doença vascular pulmonar

\begin{abstract}
This paper describes the occurrence of cor triatriatum sinister, a rare cardiac malformation in dogs, associated with pulmonary edema and pulmonary hypertension in a 5-year-old Poodle female with history of acute dyspnea and cyanosis. The animal presented acute respiratory failure, heart failure with low cardiac output, progressing to acute tubular necrosis and death. The diagnosis was made posmortem due to the clinical instability of the dog. This malformation was diagnosed by the subdivision of the left atrium into two compartments separated by an abnormal fibromuscular membrane, absence of structural abnormalities of the mitral valve and thickening of pulmonary artery tunica media associated with renal tubular degeneration. The occurrence of cor triatriatum in dogs is most common in the right atrium, defined as cor triatriatum dexter. Additionally, pulmonary arterial hypertension associated with this malformation is described only in humans with this heart defect.
\end{abstract}

Keywords: congenital heart defect, left atrium, pulmonary vascular disease

\section{REFERÊNCIAS}

ADIN, D.B.; THOMAS, W.P. Balloon dilation of cor triatriatum dexter in a dog. J. Vet. Int. Med., v.13, p.617-619, 1999.

AKINTUNDE, A.A. Cor triatriatum in an 86year-old woman: initial presentation with pulmonary hypertension discovered during preoperative evaluation. Singapore Med. J. v.10, p.203-205, 2011.

ALMEIDA, G.L.G.; ALMEIDA, M.B.; SANTOS, A.C. et al. Cor triatriatum sinister in a french bulldog. Case Rep. in Vet. Med., v.2012, p. 1-4, 2012.
BOON, A.J. Congenital Heart Disease. In: Manual of veterinary echocardiography. Willians \& Wilkins, 1998. 583p.

JOHNSON, M.S.; MARTIN, M.; DE GIOVANNI, J.V. et al. Management of cor triatriatum dexter by balloon dilatation in three dogs. J. Small Anim. Pract., v.45, p.16-20, 2004.

KITTLESON, M.D. Other congenital cardiovascular abnormalities. In: KITTLESON, M.D.; KIENLE, R.D. Small animal cardiovascular medicine. 1. ed. Mosby, St. Louis, 1998. p.282-296.

MITTEN, R.; EDWARDS, G.; RISHNIW, M. Diagnosis and management of cor triatriatum dexter in a Pyrenean Mountain Dog and an Akita Inu. Aust.Vet. J., v.79, p.177-180, 2001. 


\section{Champion et al.}

NASSAR， P.N.; HAMDAN， R.H. Cor triatriatum sinister: classification and imaging modalities. Euro. J. Cardiovasc. Med., v.1, p.8487, 2011.

OLIVEIRA, P.; DOMENECH, O.; SILVA, J. et al. Retrospective review of congenital heart disease in 976 dogs. J. Vet. Int. Med., v.25, p.477-483. 2011.

TANAKA, R.; HOSCHI, K.; SHIMIZU, M. et al. Surgical correction of cor triatriatum dexter in a dog under extracorporeal circulation. J. Small Anim. Pract., v.44, p.370-373, 2003.
THAKRAR, A.; SHAPIRO, M.D.; JASSAL D.S. et al. Cor triatriatum: the utility of cardiovascular imaging. Can. J. Cardiol., v.23, p.143-145, 2007.

TOBIAS, A.H.; THOMAS, W.P.; KITTLESON, M.D. et al. Cor triatriatum dexter in two dogs. J. Am. Vet. Med. Assoc., v.2, p.285-290, 1993. 